\title{
Addendum
}

\section{Addendum to: "Convergence towards asymptotic state in 1-D mappings: A scaling investigation” [Phys. Lett. A 379 (2015) 1246]}

\author{
Edson D. Leonel ${ }^{\mathrm{a}, \mathrm{b}, \mathrm{c}, *}$, Rivania M.N. Teixeira ${ }^{\mathrm{c}}$, Danilo S. Rando ${ }^{\mathrm{a}}$, R.N. Costa Filho ${ }^{\mathrm{c}}$, \\ Juliano A. de Oliveira ${ }^{\mathrm{d}}$ \\ a Departamento de Física, UNESP - Univ Estadual Paulista, Av. 24A, 1515 - Bela Vista, 13506-900, Rio Claro, SP, Brazil \\ b Abdus Salam International Center for Theoretical Physics, Strada Costiera 11, 34151 Trieste, Italy \\ c Departamento de Física, UFC - Univ. Federal do Ceará, Fortaleza, Ceará, Brazil \\ ${ }^{\mathrm{d}}$ UNESP - Univ Estadual Paulista, Câmpus de São João da Boa Vista, São João da Boa Vista, SP, Brazil
}

\section{A R T I C L E I N F O}

\section{Article history:}

Received 10 April 2015

Received in revised form 30 April 2015

Accepted 3 May 2015

Available online 5 May 2015

Communicated by C.R. Doering

\section{Keywords:}

Scaling law

Critical exponents

Homogeneous function

\begin{abstract}
A B S T R A C T
An analytical description of the convergence to the stationary state in period doubling bifurcations for a family of one-dimensional logistic-like mappings is made. As reported in [1], at a bifurcation point, the convergence to the fixed point is described by a scaling function with well defined critical exponents. Near the bifurcation, the convergence is characterized by an exponential decay with the relaxation time given by a power law of $\mu=R-R_{c}$ where $R_{c}$ is the bifurcation parameter. We found here the exponents $\alpha, \beta, z$ and $\delta$ analytically, confirming our numerical simulations shown in [1].
\end{abstract}

(C) 2015 Elsevier B.V. All rights reserved.
In a recent paper [1], Phys. Lett. A 379 (2015) 1246, the evolution towards the equilibrium was investigated near three types of bifurcations for a family of one-dimensional logistic-like mappings: (a) transcritical; (b) pitchfork and (c) period-doubling. It was shown that, at a bifurcation point, the convergence towards the asymptotic state is described by a generalized homogeneous function with three well defined critical exponents, namely: $\alpha, \beta$ and $z$. The exponent $\alpha$ is related with the plateau where the initial condition is temporary stationary and is heading the initial condition, $x(n) \propto x_{0}^{\alpha}$ for $n \ll n_{x}$. The exponent $\beta$ is a dynamical exponent and marks the evolution towards the stationary state. It is an algebraic function of the number of iterations of the mapping obtained from $x(n) \propto n^{\beta}$, for $n \gg n_{x}$. Finally, the changeover from one regime to the other is marked by a crossover exponent given by $n_{x} \propto x_{0}^{z}$. The exponents $\beta$ and $z$ were proved to be dependent on the nonlinearity of the mapping while $\alpha$ was universal. All the three critical exponents are related among them by a scaling law of the type $z=\alpha / \beta$.

Near the bifurcation, the evolution towards the fixed point is given by an exponential function of both $\mu=R-R_{c}$, where $R_{c}$ is

DOI of original article: http://dx.doi.org/10.1016/j.physleta.2015.02.019.

* Corresponding author. Tel.: +55 1935269174.

E-mail address: edleonel@rc.unesp.br (E.D. Leonel). the bifurcation parameter and $n$, hence $x(n) \propto e^{-n / \tau}$ where $\tau \propto \mu^{\delta}$. The exponent $\delta$ was obtained to be independent of the bifurcation and on the nonlinearity of the mapping, being fixed as $\delta=-1$.

In this Addendum, we complement the description made in Ref. [1] and give a mathematical description of how such critical exponents $\alpha, \beta$ and $z$, as well as $\delta$ appear in the period doubling bifurcations for a family of logistic-like mappings.

The family of one-dimensional logistic-like mappings, as made in Ref. [1], is given by $x_{n+1}=F\left(R, x_{n}\right)=R x_{n}\left(1-x_{n}^{\gamma}\right)$ where $\gamma \geq 1, R$ is a control parameter and $x$ is a dynamical variable. The fixed point which suffers a period doubling bifurcation is $x^{*}=[1-1 / R]^{1 / \gamma}$. The parameter $R_{c}$ where the bifurcation happens is obtained from $\left.\frac{d F}{d x}\right|_{x^{*}}=R-(1+\gamma) R x^{\gamma}=-1$, which marks a flip incipient point, leading to $R_{C}=\frac{2+\gamma}{\gamma}$.

At a period doubling bifurcation, the periodic orbits are obtained from the second iteration of the mapping, leading to the expression

$x_{n+2}=F_{R}^{(2)}\left(x_{n}\right)=R\left[R x_{n}\left(1-x_{n}^{\gamma}\right)\right]\left[1-\left[R x_{n}\left(1-x_{n}^{\gamma}\right)\right]^{\gamma}\right]$.

The idea is to expand Eq. (1) in Taylor series around the bifurcation point for the variables $x_{n}=x^{*}+y_{n}$ and $R=R_{c}+\mu$, where $y_{n}$ corresponds to the distance from the fixed point at the iterated $n$th and $\mu$ is the distance, in the control parameter, to where the 

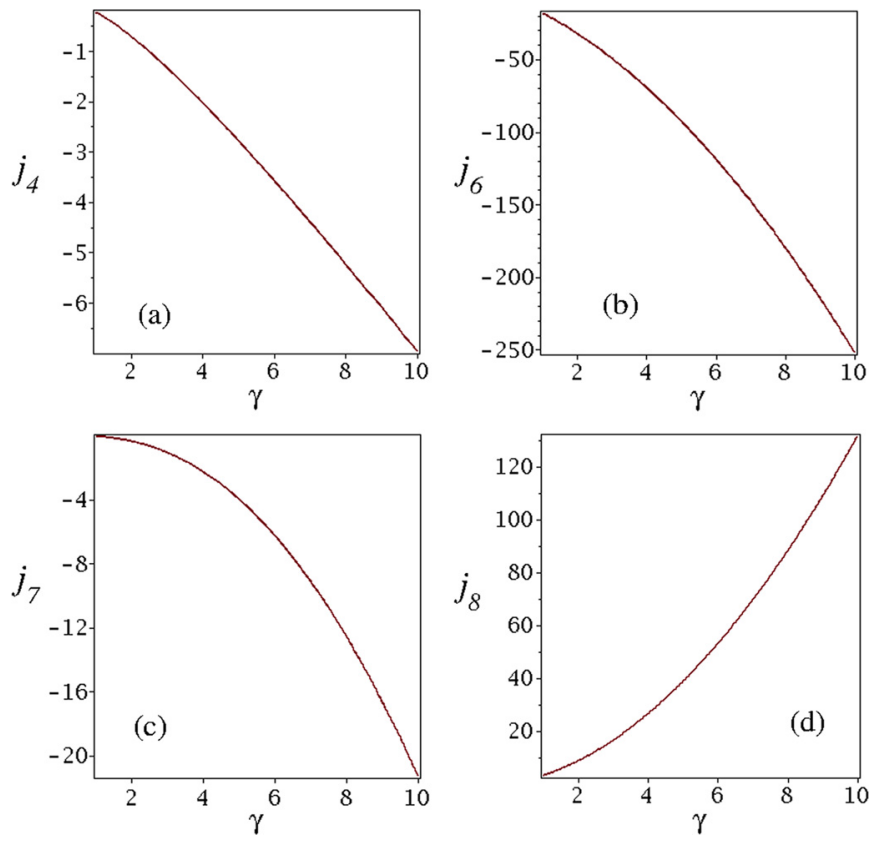

Fig. 1. Plot of the behaviour of $j_{4}, j_{6}, j_{7}$ and $j_{8}$ as a function of $\gamma$.

bifurcation happens. Hence we obtain

$$
\begin{aligned}
F_{R}^{(2)}\left(x_{n}\right) & =j_{0}+\left(x_{n}-x^{*}\right) j_{1}+\left(R-R_{c}\right) j_{2}+\left(x_{n}-x^{*}\right)^{2} j_{3} \\
& +\left(R-R_{c}\right)^{2} j_{4}+\left(x_{n}-x^{*}\right)\left(R-R_{c}\right) j_{5}+ \\
& +\left(x_{n}-x^{*}\right)^{3} j_{6}+\left(R-R_{c}\right)^{3} j_{7} \\
& +\left(x_{n}-x^{*}\right)^{2}\left(R-R_{c}\right) j_{8} \\
& +\left(x_{n}-x^{*}\right)\left(R-R_{c}\right)^{2} j_{9}+\ldots,
\end{aligned}
$$

where the auxiliary terms are

$$
\begin{aligned}
& j_{0}=F_{R_{c}}^{(2)}\left(x^{*}\right)=\left[\frac{2}{2+\gamma}\right]^{\frac{1}{\gamma}} ; \quad j_{1}=\left.\frac{\partial F^{(2)}}{\partial x}\right|_{x^{*}, R_{c}}=1, \\
& j_{2}=\left.\frac{\partial F^{(2)}}{\partial R}\right|_{x^{*}, R_{c}}=0 ; j_{3}=\left.\frac{1}{2} \frac{\partial^{2} F^{(2)}}{\partial x^{2}}\right|_{x^{*}, R_{c}}=0, \\
& j_{4}=\left.\frac{1}{2} \frac{\partial^{2} F^{(2)}}{\partial R^{2}}\right|_{x^{*}, R_{c}} ; \quad j_{5}=\left.\frac{\partial^{2} F^{(2)}}{\partial x \partial R}\right|_{x^{*}, R_{c}}=2 \gamma, \\
& j_{6}=\left.\frac{1}{6} \frac{\partial^{3} F^{(2)}}{\partial x^{3}}\right|_{x^{*}, R_{c}} ; \quad j_{7}=\left.\frac{1}{6} \frac{\partial^{3} F^{(2)}}{\partial R^{3}}\right|_{x^{*}, R_{c}}, \\
& j_{8}=\left.\frac{1}{2} \frac{\partial^{3} F^{(2)}}{\partial x^{2} \partial R}\right|_{x^{*}, R_{c}} ; \quad j_{9}=\left.\frac{1}{2} \frac{\partial^{3} F^{(2)}}{\partial x \partial R^{2}}\right|_{x^{*}, R_{c}}=\gamma^{2} .
\end{aligned}
$$

The expressions for $j_{4}, j_{6}, j_{7}$ and $j_{8}$ are extensive and do not need to be presented. Instead, their behaviour as a function of $\gamma$ allow us to collect the most information on them, as shown in Fig. 1. We can see all of them are well defined and smooth. The most important information regards, particularly, on the signal of $j_{6}$, which by now is negative. Table 1 presents some numerical values of $j_{4}, j_{6}, j_{7}$ and $j_{8}$, evaluated at the period doubling bifurcation for different values of $\gamma$. Because $j_{3}$ is zero, the Taylor expansion was made to higher order terms in $\left(x-x^{*}\right)$ to guarantee a minimal nonlinearity to the difference equation.
Table 1

Table of values for $j_{4}, j_{6}, j_{7}$ and $j_{8}$ for different values of $\gamma$.

\begin{tabular}{llllll}
\hline$\gamma$ & 1 & 2 & 3 & 4 & 5 \\
\hline$j_{4}$ & $-\frac{2}{9}$ & $-\frac{\sqrt{2}}{2}$ & $-\frac{9}{25} \sqrt[3]{50}$ & $-\frac{8}{9} \sqrt[4]{27}$ & $-\frac{25}{49} \sqrt[5]{4802}$ \\
$j_{6}$ & -18 & -32 & $-\frac{40}{3} \sqrt[3]{50}$ & $-40 \sqrt{3}$ & $-28 \sqrt[5]{392}$ \\
$j_{7}$ & $-\frac{4}{81}$ & $-\frac{\sqrt{2}}{4}$ & $-\frac{36}{125} \sqrt[3]{50}$ & $-\frac{80}{81} \sqrt[4]{27}$ & $-\frac{250}{343} \sqrt[5]{4802}$ \\
$j_{8}$ & 3 & $6 \sqrt{2}$ & $6 \sqrt[3]{20}$ & $20 \sqrt[4]{3}$ & $15 \sqrt[5]{112}$ \\
\hline
\end{tabular}

Considering then the expressions $x_{n}=x^{*}+y_{n}$ and $R=R_{C}+\mu$ and the expansion of $F_{R}^{(2)}\left(x_{n}\right)$, we can rewrite Eq. (1) as

$$
\begin{aligned}
G\left(y_{n}, \mu\right) & =F_{R}^{(2)}\left(x_{n}\right)-F_{R_{c}}^{(2)}\left(x^{*}\right), \\
& \cong y_{n}+\mu^{2} j_{4}+2 \gamma \mu y_{n}+y_{n}^{3} j_{6}+\mu^{3} j_{7} \\
& +y_{n}^{2} \mu j_{8}+y_{n} \mu^{2} \gamma^{2} .
\end{aligned}
$$

Let us start first discussing the properties of the convergence to the fixed point exactly at the bifurcation point. This leads to $\mu=0$. Hence we obtain $G\left(y_{n}, 0\right)=y_{n}+y_{n}^{3} j_{6}$. If we consider here the approximation $G\left(y_{n}, 0\right)-y_{n} \cong \frac{d y}{d n}=y^{3} j_{6}$, hence we have to integrate the following differential equation $\int_{y_{0}}^{y(n)} \frac{d y}{y^{3}}=j_{6} \int_{0}^{n} d n$. Doing the integration, appropriate simplification and considering $j_{6}$ is negative, we obtain $y(n)=\frac{y_{0}}{\sqrt{1+2\left|j_{6}\right| y_{0}^{2} n}}$. From this expression we can discuss the numerical results obtained from Ref. [1]. The first scaling comes from the condition $2\left|j_{6}\right| y_{0}^{2} n \ll 1$. Then we obtain $y(n) \propto y_{0}$. From the first scaling hypothesis presented in Ref. [1], we found $y(n) \propto y_{0}^{\alpha}$. Therefore $\alpha=1$. It is indeed universal for the family of logistic-like mapping discussed here and does not depend on $\gamma$. The second scaling is obtained from the condition $2\left|j_{6}\right| y_{0}^{2} n \gg 1$ leading to $y(n) \propto \frac{1}{\sqrt{2\left|j_{6}\right|}} n^{-1 / 2}$. A second scaling hypothesis discussed in Ref. [1] argues that $y(n) \propto n^{\beta}$. Hence we found analytically $\beta=-1 / 2$. This exponent was obtained in Ref. [1] only by numerical simulations as $\beta=-0.49939$ (7), therefore very close to our present finding validating the numerical simulation. Finally, the exponent $z$ comes from the condition $2\left|j_{6}\right| y_{0}^{2} n=1$. This is the condition leading to the changeover from the constant plateau to the regime of decay. Therefore we obtain $n_{x}=\frac{1}{2\left|j_{6}\right| y_{0}^{2}} \propto y_{0}^{-2}$. The latest scaling hypothesis discussed in Ref. [1] proposes that $n_{x} \propto y_{0}^{z}$. Numerically the result obtained was $z=-2.001$ (4), which is remarkably close to our analytical finding here.

Given the theory fits well with the numerical simulations for $\mu=0$, let us now discuss the case of $\mu \neq 0$. To do so, we shall consider only the lowest representative term of $\mu$ in Eq. (3), leading to $G\left(y_{n}, \mu\right) \cong y_{n}+2 \gamma y_{n} \mu+j_{6} y_{n}^{3}$. Transforming the equation of differences into a differential equation, we have $G\left(y_{n}, \mu\right)-y_{n} \cong \frac{d y}{d n}=$ $y\left(2 \gamma \mu+j_{6} y^{2}\right)$. The integration to be solved is $\int_{y_{0}}^{y(n)} \frac{d y}{y\left(2 \gamma \mu+j_{6} y^{2}\right)}=$ $\int_{0}^{n} d n$. Doing the integration and grouping the terms properly, we obtain

$y(n)=\sqrt{\frac{2 \mu \gamma}{\left|j_{6}\right|}}\left[1+\left(\frac{2 \mu \gamma}{y_{0}^{2}\left|j_{6}\right|}-1\right) e^{-4 \mu \gamma n}\right]^{-\frac{1}{2}}$

To have a better insight in the consequences of Eq. (4), it is convenient to expand it in Taylor series. Therefore we obtain

$y(n) \cong \sqrt{\frac{2 \mu \gamma}{\left|j_{6}\right|}}\left[1-\frac{1}{2}\left(\frac{2 \mu \gamma}{y_{0}^{2}\left|j_{6}\right|}-1\right) e^{-4 \mu \gamma n}\right]$.

Keeping only a linear power of $\mu$, hence disregarding terms of the order $\mu \sqrt{\mu}$, Eq. (5) can be written as $y(n)-\sqrt{\frac{2 \gamma \mu}{\left|j_{6}\right|}} \cong$ $\sqrt{\frac{\gamma \mu}{2\left|j_{6}\right|}} e^{-4 \mu \gamma n}$. Comparing this equation with the simulation dis- 
cussed in Ref. [1] we conclude $y(n) \propto e^{-4 \gamma \mu n}$ hence leading to $\delta=-1$, in agreement with the simulation shown in [1].

\section{Acknowledgements}

RMNT thanks to FUNCAP. RNCF thanks to CNPq. JAO thanks to CNPq, PROPe/FUNDUNESP and FAPESP (2014/18672-8). EDL ac- knowledges support from CNPq, FAPESP (2012/23688-5) and FUNDUNESP.

\section{References}

[1] R.M.N. Teixeira, D.S. Rando, F.C. Geraldo, R.N. Costa Filho, J.A. de Oliveira, E.D Leonel, Phys. Lett. A 379 (2015) 1246. 